\title{
HDAC1 promotes the migration of human myeloma cells via regulation of the IncRNA/Slug axis
}

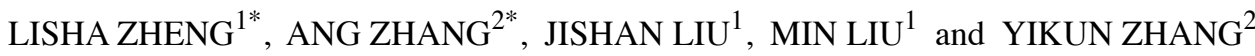 \\ ${ }^{1}$ School of Biological Science and Medical Engineering, Beihang University, Beijing 100191; ${ }^{2}$ Department of Hematology, \\ PLA Strategic Support Force Characteristic Medical Center, Beijing 100101, P.R. China
}

Received April 3, 2021; Accepted September 21, 2021

DOI: $10.3892 /$ ijmm.2021.5058

\begin{abstract}
Understanding the mechanisms underlying malignancy in myeloma cells is important for targeted treatment and drug development. Histone deacetylases (HDACs) can regulate the progression of various cancer types; however, their roles in myeloma are not well known. In the present study, the expression of class I HDACs in myeloma cells and tissues was evaluated. Furthermore, the effects of HDAC1 on the migration of myeloma cells and the associated mechanisms were investigated. Among the class I HDACs evaluated, HDAC1 was upregulated in both myeloma cells and tissues. Targeted inhibition of HDAC1 suppressed the migration of myeloma cells. Of the assessed transcription factors, small interfering (si)-HDAC1 decreased the expression of Slug. Overexpression of Slug reversed the si-HDAC1-mediated suppressed migration of myeloma cells. Mechanistically, the results revealed that $\mathrm{HDAC1}$ regulated the mRNA stability of Slug, while it had no effect on its transcription or nuclear export. Furthermore, HDAC1 negatively regulated the expression of long non-coding RNA (IncRNA) NONHSAT113026, which could bind with the 3'-untranslated region of Slug mRNA to facilitate its degradation. The present study demonstrated that HDAC1 promoted the migration of human myeloma cells via regulation of lncRNA/Slug signaling.
\end{abstract}

\section{Introduction}

Multiple myeloma (MM) is a hematological malignancy that is characterized by the abnormal expansion of monoclonal plasma cells in the bone marrow (1). MM is the most

Correspondence to: Professor Yikun Zhang, Department of Hematology, PLA Strategic Support Force Characteristic Medical Center, 9 Anxiang North Lane, Deshengmen, Chaoyang, Beijing 100101, P.R. China

E-mail: yikunzhang@protonmail.com

\section{*Contributed equally}

Key words: HDAC1, myeloma, Slug, long non-coding RNA, migration common hematological cancer worldwide, and it accounts for $\sim 13 \%$ of all hematological malignancies (2). Various therapy approaches, including chemotherapy, autologous/allogeneic stem cell transplantation and monoclonal antibodies, have been developed for MM treatment $(3,4)$. However, the clinical therapeuric efficiency of MM remains unsatisfactory (5-7). E-cadherin (E-Cad) protein, an inhibitor of epithelial-mesenchymal transition (EMT), was reported to be decreased in metastatic MM tissues, while the expression of Slug, MMP-2 and MMP-9 proteins was increased (8). Therefore, research on the mechanisms underlying MM development and cell migration are required to improve patient outcomes.

Genetic and epigenetic abnormalities, such as chromosomal translocations and histone acetylation, are common features of MM (9-12). Histone deacetylases (HDACs) can remove acetyl groups from $\mathrm{N}$-acetyllysines on histones to regulate the progression of various cancer types, including MM (13). Targeted inhibition of HDAC can induce cell cycle arrest and apoptosis of cancer cells (14). Inhibitors of HDAC, such as butyrate, trichostatin A and suberoylanilide hydroxamic acid, have been considered as candidate drugs for cancer therapy $(15,16)$. Regarding MM, it has been reported that inhibition of HDAC6 can increase the expression of CD38 and augment the efficacy of daratumumab in MM cells (17). Peptides that inhibit HDAC can overcome bortezomib resistance in MM (18). Nexturastat A, the selective inhibitor of HDAC6, can induce apoptosis, overcome drug resistance and inhibit tumor growth in MM (19). Overall, these data suggest that HDACs may play important oncogenic roles in MM development.

Aberrant overexpression of class I HDACs (HDAC1, 2, 3 and 8) has been observed in MM tissues (20). In addition, class-I HDACs may serve more important roles in cancer progression than other HDACs, among the 18 HDAC enzymes identified in humans $(21,22)$. However, to the best of our knowledge, no attempt has been made thus far to explain the roles and underlying mechanisms of class I HDACs in MM development.

The aim of the present study was to investigate whether HDAC1 can positively regulate the migration of MM cells, and to perform mechanistic studies in order to determine whether HDAC1 promotes the malignancy of human MM cells via regulation of long non-coding RNA (lncRNA) NONHSAT113026/Slug signaling. 


\section{Materials and methods}

Cell lines and cell culture. Human MM U266, RPMI8226 and H929 cells were purchased from The Cell Bank of Type Culture Collection of The Chinese Academy of Sciences. The human normal plasma cell line (nPCs) was acquired from $\mathrm{BeNa}$ Culture Collection (cat. no. BNCC350752; http://www.bncc.org. $\left.\mathrm{cn} / \mathrm{pro} / \mathrm{p1} / 5 / \mathrm{p} \_943 . \mathrm{html}\right)$. All cells were cultured in RPMI-1640 medium (HyClone; Cytiva) supplemented with 10\% FBS (FBS; cat. no. S601P-500; Sera Pro), 1\% L-glutamine and 1\% penicillin/streptomycin in humidified air with $5 \% \mathrm{CO}_{2}$ at $37^{\circ} \mathrm{C}$.

$R N A$ extraction and reverse transcription-quantitative $P C R$ ( $R T$ - $q P C R)$. Total RNA from human MM cells was extracted using TRIzol ${ }^{\circledR}$ (Thermo Fisher Scientific, Inc.) following the manufacturer's instructions. After isolation, $500 \mathrm{ng}$ total RNA was used to generate complementary DNA using PrimeScript RT Reagent kit (Takara Biotechnology Co., Ltd.) at room temperature according to the manufacturer's protocol. qPCR was performed using SYBR Green PCR kit (Applied Biosystems; Thermo Fisher Scientific, Inc.) and analyzed with MyiQ Real Time RT-PCR System (Bio-Rad Laboratories, Inc.). The PCR cycling conditions were as follows: $15 \mathrm{~min}$ at $95^{\circ} \mathrm{C}$, followed by 40 cycles for $10 \mathrm{sec}$ at $95^{\circ} \mathrm{C}, 30 \mathrm{sec}$ at $60^{\circ} \mathrm{C}$ and $1 \mathrm{sec}$ at $72^{\circ} \mathrm{C}$, and 1 cycle of cooling for $30 \mathrm{sec}$ at $50^{\circ} \mathrm{C}$.

The primer sequences used in the present study were as follows: HDAC1 forward, 5'-CTACTACGACGGGGATGT TGG-3' and reverse 5'-GAGTCATGCGGATTCGGTGAG-3'; HDAC2 forward, 5'-ATGGCGTACAGTCAAGGAGG-3' and reverse, 5'-TGCGGATTCTATGAGGCTTCA-3'; HDAC3 forward, 5'-CCTGGCATTGACCCATAGCC-3' and reverse 5'-CTCTTGGTGAAGCCTTGCATA-3'; HDAC8 forward, 5'-TCGCTGGTCCCGGTTTATATC-3' and reverse, 5'-TAC TGGCCCGTTTGGGGAT-3'; MMP-2 forward, 5'-TACAGG ATCATTGGCTACACACC-3' and reverse, 5'-GGTCACATC GCTCCAGACT-3'; MMP-9 forward, 5'-TGTACCGCTATG GTTACACTCG-3' and reverse, 5'-GGCAGGGACAGTTGC TTCT-3'; E-Cad forward, 5'-CGAGAGCTACACGTTCAC GG-3' and reverse, 5'-GGGTGTCGAGGGAAAAATAGG-3'; Snail forward, 5'-TCGGAAGCCTAACTACAGCGA-3' and reverse, 5'-AGATGAGCATTGGCAGCGAG-3'; Slug forward, 5'-AGATGCCGCGCTCCTTCCTG-3' and reverse, 5'-GCA AACGAAGCTGCGAGATT-3'; zinc finger E-box binding homeobox 1 (Zeb1) forward, 5'-GATGATGAATGCGAG TCAGATGC-3' and reverse, 5'-ACAGCAGTGTCTTGT TGTTGT-3'; Twist forward, 5'-GTCCGCAGTCTTACGAGG AG-3' and reverse, 5'-GCTTGAGGGTCTGAATCTTGCT-3'; NONHSAT forward, 5'-TACACCTGGTTTCCTCCCTCA T-3' and reverse, 5'-AAAGTGCCCCAAATCTTTGTTC-3'; and GAPDH forward, 5'-TTCCAGGAGTGAGTGGAAGA-3' and reverse, 5'-GCAGAGAAGCAGACAGTTATG-3'. The expression of each gene was normalized to the endogenous reference GAPDH via the $2^{-\triangle \Delta C q}$ method (23). Experiments were performed in triplicate.

Western blot analysis. Cells were lysed using RIPA buffer (Santa Cruz Biotechnology, Inc.) supplemented with a protease inhibitor cocktail (Roche Applied Science). Protein concentration was determined using a NanoDrop ND-1000 UV-Vis Spectrophotometer (NanoDrop Technologies; Thermo Fisher
Scientific, Inc.). Next, $30 \mu \mathrm{g}$ total protein from each lysate was separated using 10 or $12 \%$ SDS-PAGE and subsequently electroblotted onto a PVDF membrane. The membranes were blocked with $5 \%$ skimmed milk at room temperature for $2 \mathrm{~h}$. The target proteins were probed with primary antibodies $(1: 1,000)$ overnight at $4^{\circ} \mathrm{C}$ and further incubated with HRP-conjugated secondary antibody (cat. no. ab7090; Abcam; 1:10,000). The primary antibodies were specific for GAPDH (cat. no. ab181602; Abcam), HDAC1 (cat. no. ab53091; Abcam), HDAC2 (cat. no. ab7029; Abcam), HDAC3 (cat. no. ab96005; Abcam), HDAC8 (cat. no. 17548-1-AP; ProteinTech Group, Inc.), MMP-2 (cat. no. ab97779; Abcam), MMP-9 (cat. no. PA5-27191; Thermo Fisher Scientific, Inc.), E-cad (cat. no. ab231303; Abcam) and Slug (cat. no. ab27568; Abcam). GAPDH was used as the loading control for normalization. The signals were detected by enhanced chemiluminescence using a Chemidoc XRS Molecular Imager (Bio-Rad Laboratories Inc.). Quantity One software (version 4.3.0; Bio-Rad Laboratories, Inc.) was used for densitometric analysis.

Cell transfection. Cells were seeded in 6-well plates at $50-60 \%$ confluence. Cell transfection was conducted using Lipofectamine ${ }^{\circledR} 3000$ reagent (Invitrogen; Thermo Fisher Scientific, Inc.) according to the manufacturer's instructions at room temperature. The time interval between transfection and subsequent experimentation was $24 \mathrm{~h}$.

Specific small interfering (si)RNA for HDAC1 (si-HDAC1-1, 5'-GAGUCAAAACAGAGGAUGA-3'; si-HDAC1-2, 5'-AAC TATGGTCTC TACCGAAAA-3'), NONHSAT 113026 (5'-GCGCCACACTGGCCCGCGCCA-3'), and negative control (NC) siRNA (si-NC; 5'-GCACAACAAGCCGAA UACA-3') were purchased from Guangzhou RiboBio Co., Ltd. The working concentration for siRNA was $25 \mathrm{nM}$ when cells were transfected.

For overexpression, the sequence of Slug was cloned into the pcDNA vector (Invitrogen; Thermo Fisher Scientific, Inc.) to construct the overexpression vector pcDNA/Slug, and empty pcDNA was used as the NC. Cells seeded in 6-well plates at $\sim 60 \%$ confluence were transfected with vector control, plasmid constructs (final concentration of $20 \mu \mathrm{g} / \mathrm{ml}$ ) or siRNAs (final concentration of $20 \mathrm{nmol} / \mathrm{l}$ ). The transfection efficiency was evaluated by western blot analysis and/or RT-PCR.

Wound healing assay. Cells were transiently transfected with siRNA or plasmid as aforementioned. Straight lines were drawn vertically on the back of a 12 -well plate before seeding the cells. When the cells reached $90 \%$ confluence, the cell monolayer was gently scratched with a $100-\mu 1$ pipette tip according to the marked lines and washed with PBS three times. Next, RPMI-1640 medium (HyClone; Cytiva) without FBS was added to the plate. The imaging position was recorded at 0 and $24 \mathrm{~h}$ by using an inverted microscope (Leica Microsystems $\mathrm{GmbH}$; magnification, $\mathrm{x} 10)$. The results were determined by calculating the area occupied by migrated cells at five random areas for each wound as follows: (Original wound width at $0 \mathrm{~h}$-actual wound width at $24 \mathrm{~h}$ )/(original wound width at $0 \mathrm{~h}$ ).

Luciferase assays. The luciferase reporter construct pGL-Slug was generated by inserting the promoter of Slug $(-1,000$ to $+1 \mathrm{bp})$ according to a previous study (24). The dual-luciferase 
expression plasmids pGL-Slug and pGL3-IE1-Rluc (50 ng, Promega Corporation) were co-transfected into cells. After $\sim 24 \mathrm{~h}$, luciferase activity was determined with Dual-Glo Luciferase Assay kit (Promega Corporation) according to the manufacturer's instructions by using a multilabel plate reader (Wallac VICTOR; PerkinElmer, Inc.). The relative luciferase activity (firefly luciferase/Renilla luciferase) was calculated. Each experiment was repeated three times.

Cell nuclear and cytoplasmic RNA isolation. Nuclear and cytoplasmic RNA were extracted from U266 cells using the PARIS kit (Thermo Fisher Scientific, Inc.) and TRIzol ${ }^{\circledR}$ (Thermo Fisher Scientific, Inc.), according to a previous study (25).

$m R N A$ stability assay. Cells were pre-transfected with si-NC or si-HDAC1 for $24 \mathrm{~h}$ and then further treated with $5 \mu \mathrm{g} / \mathrm{ml}$ actinomycin D (Act-D; cat. no. A9415; Sigma-Aldrich; Merck KGaA) for 0 to $8 \mathrm{~h}$. Total RNA was extracted by using TRIzol ${ }^{\circledR}$, and the levels of Slug were analyzed by using RT-qPCR, as aforementioned. The levels of Slug were normalized to those of GAPDH.

Oncomine database analysis. The online microarray database Oncomine $^{\mathrm{TM}}$ was used to explore the mRNA expression levels of HDAC1 in MM and adjacent normal tissues (https://www. oncomine.org/resource/login.html). The conditions for filter settings were as follows: Gene, 'HDAC1'; Cancer Type, 'Myeloma'.

Statistical analysis. Data are presented as the mean \pm standard deviation from $\geq 3$ separate experiments. Statistical differences were determined by performing an unpaired two-tailed Student's t-tests and one-way ANOVA followed by Bonferroni's post hoc test using SPSS 15.0 (SPSS, Inc.). GraphPad Prism 8.0 software (GraphPad Software, Inc.) was used to represent data on graphs. $\mathrm{P}<0.05$ was considered to indicate a statistically significant difference.

\section{Results}

HDACl is increased in MM cells and tissues. Firstly, the mRNA and protein levels of class I HDACs in MM U266, RPMI8226 and NCI-H929 cells, and in nPCs cells were determined. RT-qPCR revealed that the expression of HDAC1, but not of HDA2, -3 or -8 , was significantly upregulated in MM cells compared with that in nPCs cells (Fig. 1A). Furthermore, western blot analysis also confirmed the upregulation of HDAC1 in MM cells (Fig. 1B).

The present study also evaluated the expression of HDAC1 in MM and normal tissues with data from the Oncomine database. The results revealed a significantly increased expression of HDAC1 in MM tissues compared with that in adjacent normal tissues according to the data reported by Talantov (Fig. 1C) and Haqq (Fig. 1D) on myeloma. Overall, these data indicated that HDAC1 was upregulated in MM cells and tissues.

HDAC1 regulates the migration of MM cells. In order to evaluate the potential roles of HDAC1 in the progression of MM, human MM U266 and RPMI8226 cells were transfected with
HDAC1 siRNA(Fig. 2A). The data showed that si-HDAC1 could significantly decrease the migration of U266 and RPMI8226 cells, compared with the si-NC (Fig. 2B). RT-qPCR revealed that si-HDAC1 treatment decreased the mRNA expression of MMP-2 and MMP-9, as well as increasing the expression of E-Cad, in U266 (Fig. 2C) and RPMI8226 (Fig. 2D) cells.

Western blot analysis confirmed that si-HDAC1 decreased the expression of MMP-2 and MMP-9, and increase the expression of E-Cad, in U266 cells (Fig. 2E), which indicated that HDAC1 regulated the migration of MM cells.

Slug is essential for the HDAC1-regulated motility of MM cells. Transcription factors such as Snail, Slug, Zeb1 and Twist, which can trigger the EMT of cancer cells, are critical for the motility of cancer cells $(26,27)$.

The present study found that si-HDAC1 significantly decreased the mRNA expression level of Slug, but not that of Snail, Zeb1 or Twist, in U266 (Fig. 3A) and RPMI8226 (Fig. 3B) cells. Western blot analysis confirmed that si-HDAC1 decreased the protein expression of Slug in U266 and RPMI8226 cells (Fig. 3C).

The effects of Slug on the si-HDAC1-suppressed migration of U266 cells were evaluated via overexpression of Slug (Fig. 3D). Migration assay showed that overexpression of Slug could reverse the si-HDAC1-suppressed migration of U266 cells (Fig. 3E). In addition, overexpression of Slug also attenuated the si-HDAC1-increased expression of E-Cad in U266 cells (Fig. 3F). These data revealed that Slug was essential for the HDAC1-mediated regulation of motility of MM cells.

HDAC1 positively regulates the $m R N A$ stability of Slug. The mechanism underlying the HDAC1-regulated expression of Slug was next investigated. The present data indicated that si-HDAC1 had no effect on the precursor mRNA expression of Slug in either U266 or RPMI8226 cells (Fig. 4A).

Furthermore, the promoter of Slug $(-1,000$ to +1$)$ was subcloned in the pGL3 luciferase reporter. Dual-luciferase assay showed that si-HDAC1 had no effect on the promoter activity of Slug in MM cells (Fig. 4B).

By separating nuclear and cytoplasmic RNA, RT-qPCR analysis showed that si-HDAC1 had no effect on the nuclear export of Slug in MM cells (Fig. 4C). However, si-HDAC1 could significantly decrease the mRNA stability of Slug in both U266 (Fig. 4D) and RPMI8226 (Fig. 4E) cells. These results indicated that HDAC1 could positively regulate the mRNA stability of Slug.

HDAC1 negatively regulates the expression of lncRNA-NONHSAT113026 in MM cells. It has been reported that IncRNA can bind to mature mRNA to regulate mRNA stability in cancer cells $(28,29)$. IncRNA NONHSAT113026 can bind the 3'-untranslated region (UTR) of Slug via direct lncRNA-mRNA interactions, which results in the degradation of mRNA (30). The present results showed that knockdown of HDAC1 significantly increased the expression of lncRNA NONHSAT113026 in U266 and RPMI8226 cells (Fig. 5A).

Furthermore, knockdown of lncRNA NONHSAT113026 was successfully performed (Fig. 5B) and this resulted in increased migration of U266 cells (Fig. 5C). In addition, knockdown of IncRNA NONHSAT113026 could increase 

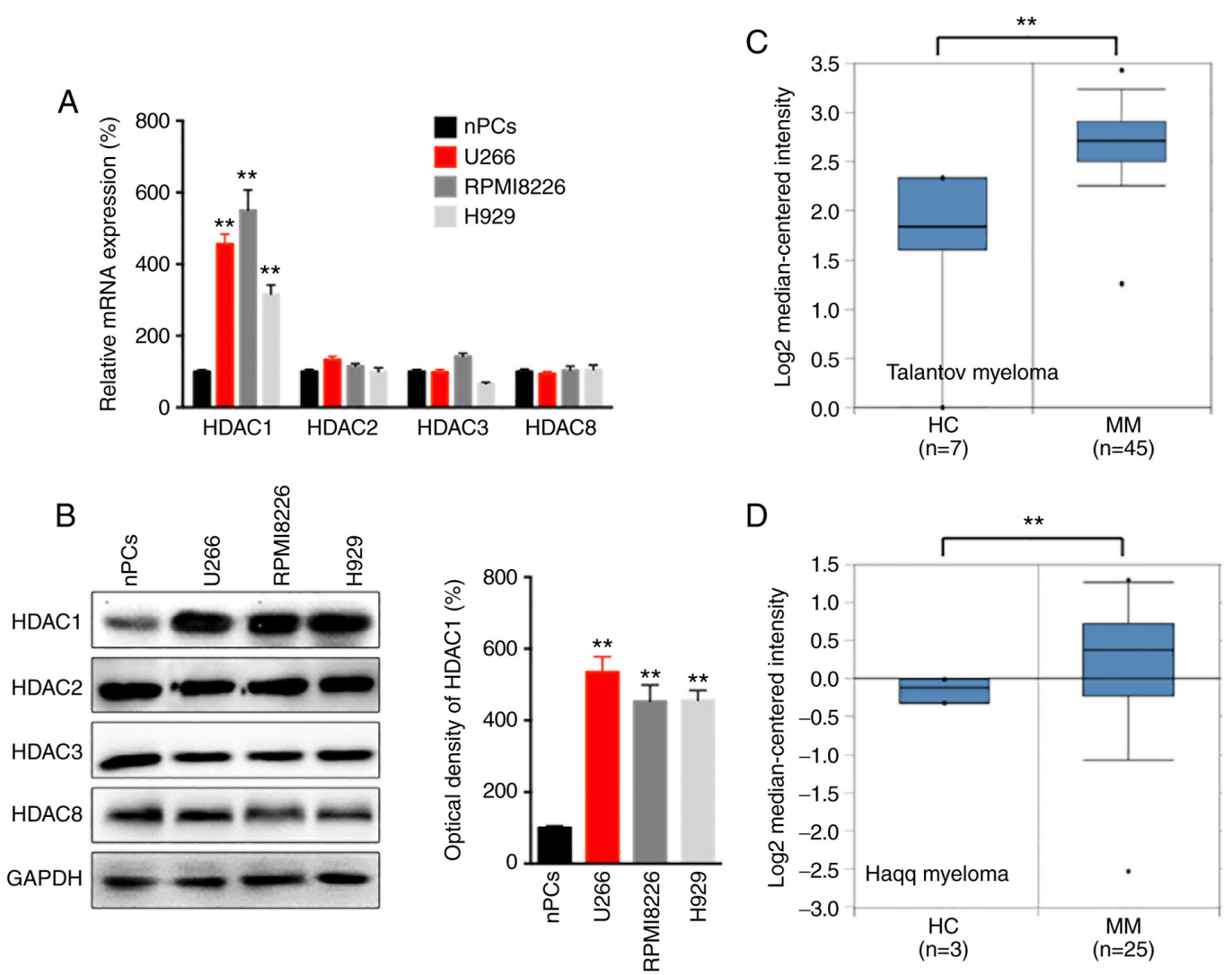

Figure 1. HDAC1 is increased in MM cells and tissues. (A) The mRNA expression of class I HDACs in MM and nPCs cells were evaluated. (B) The protein expression of HDACs in MM and nPCs cells were determined. Expression of HDAC1 in human MM cancer tissues and normal mucosa tissues from (C) Talantov and (D) Haqq myeloma datasets from the Oncomine database. Data are presented as mean \pm SD of three independent experiments. ${ }^{* *} \mathrm{P}<0.01$. HDAC1, histone deactylase 1; MM, multiple myeloma; nPCs, normal plasma cells.

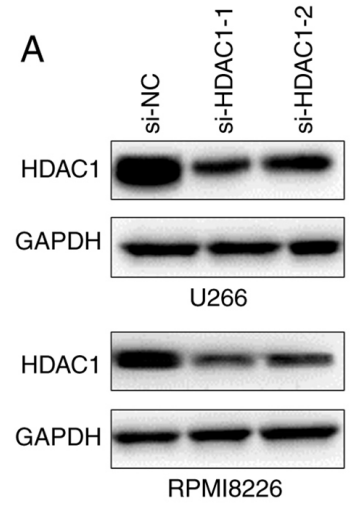

C

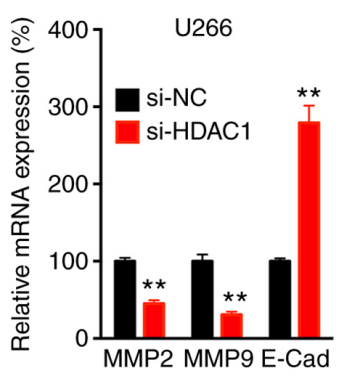

B
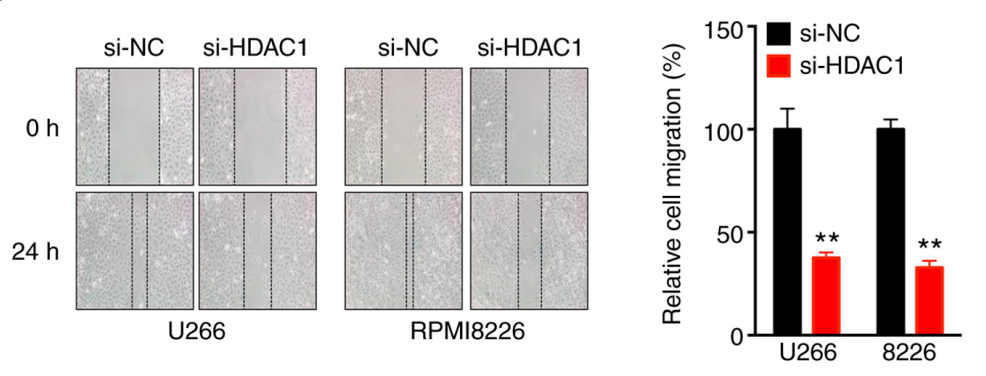

$\mathrm{D}$

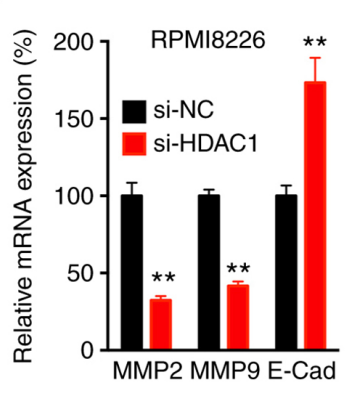

$\mathrm{E}$

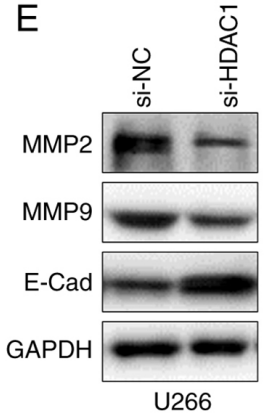

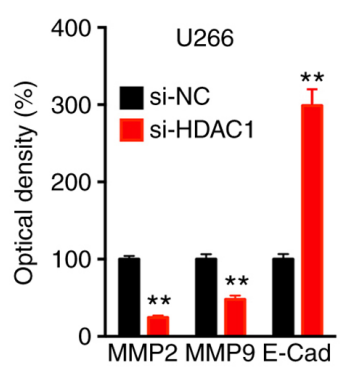

Figure 2. HDAC8 regulates the migration of MM cells. (A) Cells were transfected with si-NC or si-HDAC1-1 or -2 for $24 \mathrm{~h}$, the protein expression of HDAC1 was checked, si-HDAC1-1 was used for subsequent experiments. (B) Wound healing assay for cells transfected with si-NC or si-HDAC1 for $24 \mathrm{~h}$. (C) U266 and (D) RPMI8226 cells were transfected with si-NC or si-HDAC1 for $24 \mathrm{~h}$, the mRNA expression of MMP2, MMP9 and E-Cad was evaluated. (E) U266 cells were transfected with si-NC or si-HDAC1 for $24 \mathrm{~h}$, the protein expression of MMP2, MMP9 and E-Cad was determined and quantitatively analyzed. Data are presented as the mean $\pm \mathrm{SD}$ of three independent experiments. ${ }^{* *} \mathrm{P}<0.01$. HDAC1, histone deactylase $1 ; \mathrm{MM}$, multiple myeloma; si, small interfering; NC, negative control; E-Cad, E-Cadherin. 
A
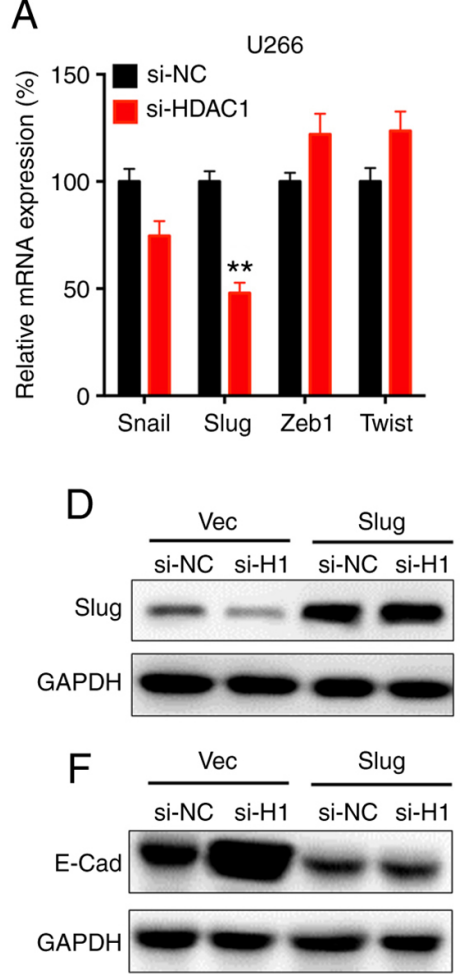

B

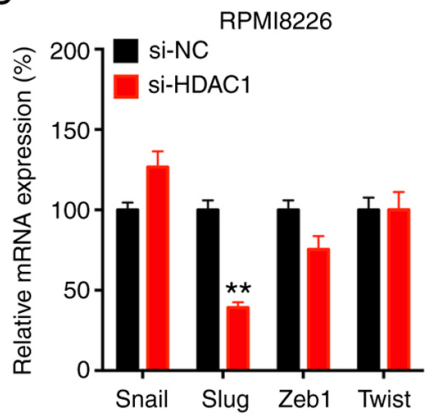

$\mathrm{E}$

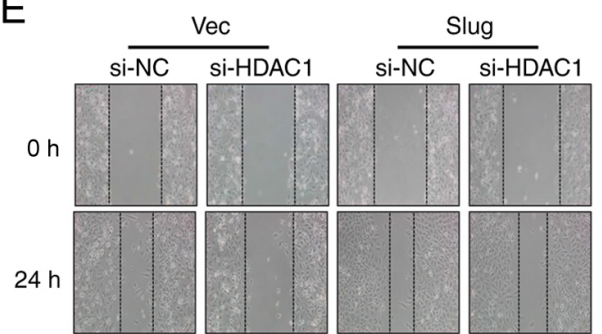

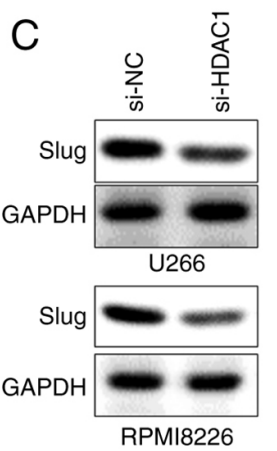
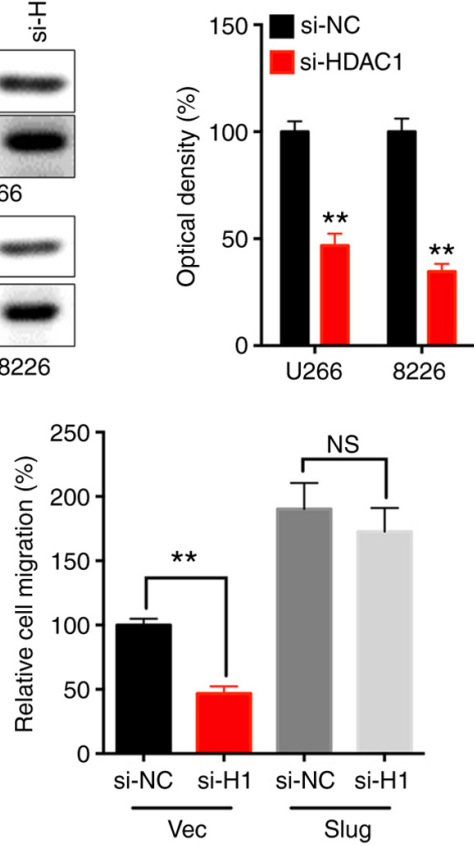

Figure 3. Slug is essential for HDAC1-regulated motility of MM cells. (A) U266 and (B) RPMI8226 cells were transfected with si-NC or si-HDAC1 for 24 h, the mRNA expression was determined via RT-qPCR. (C) Cells were transfected with si-NC or si-HDAC1 for $24 \mathrm{~h}$, the protein expression of Slug was checked. U266 cells were co-transfected with si-NC, si-HDAC1, vector control or pcDNA/Slug for $24 \mathrm{~h}$. (D) Dxpression of Slug was determined by western blot analysis. (E) Migration was assessed by wound healing assay. (F) the expression of E-Cad was checked by western blot analysis. Data are presented as the mean \pm SD of three independent experiments. " $\mathrm{P}<0.01$, NS, not significant; HDAC1, histone deactylase 1; MM, multiple myeloma; si, small interfering; NC, negative control; Vec, vector.

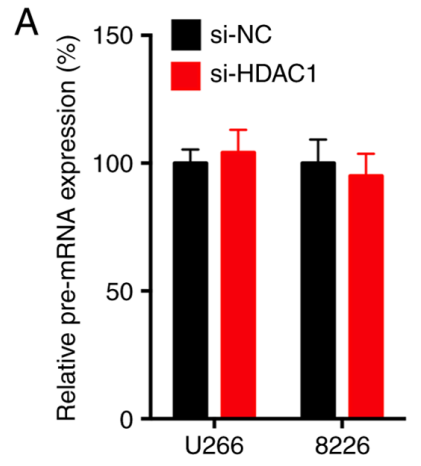

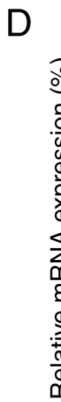

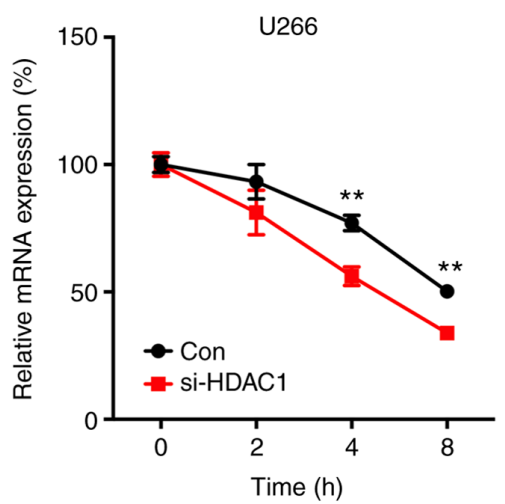

B

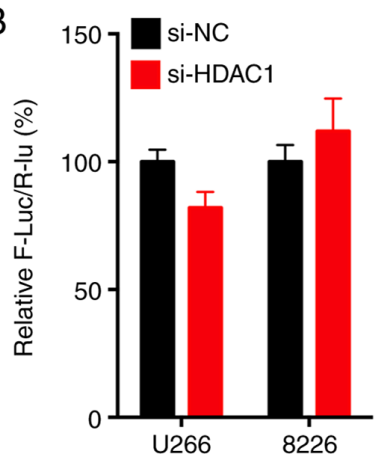

C

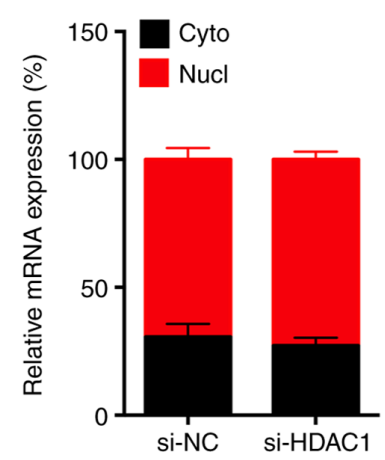

RPMI8226
$\mathrm{E}$

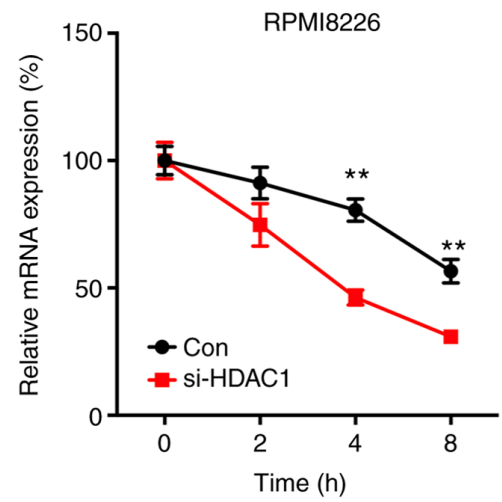

Figure 4. HDAC1 positively regulates the mRNA stability of Slug. (A) Cells were transfected with si-NC or si-HDAC1 for $24 \mathrm{~h}$, the precursor mRNA of Slug was determined by RTq-PCR. (B) Cells were co-transfected with si-NC, si-HDAC1 or pGL3-Slug for $24 \mathrm{~h}$, the promoter activity was evaluated via dual-luciferase assay. (C) Total RNA from the cytoplasm and nucleic fraction of U266 cells transfected with si-NC or si-HDAC1 for $24 \mathrm{~h}$ were isolated, the levels of Slug mRNA was measured. (D) U266 or (E) RPMI8226 cells were pre-transfected with si-NC or si-HDAC1 for $24 \mathrm{~h}$ and then further treated with Act-D for the indicated time periods before the mRNA expression of Slug was measured. Data are presented as the mean $\pm \mathrm{SD}$ of three independent experiments. ${ }^{* *} \mathrm{P}<0.01$. HDAC1, histone deactylase 1; MM, multiple myeloma; si, small interfering; NC, negative control; Cyto, cytoplasm; Nucl, nucleus; Con, control. 
A

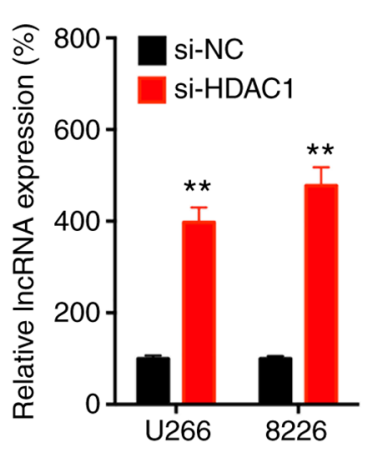

$\mathrm{B}$

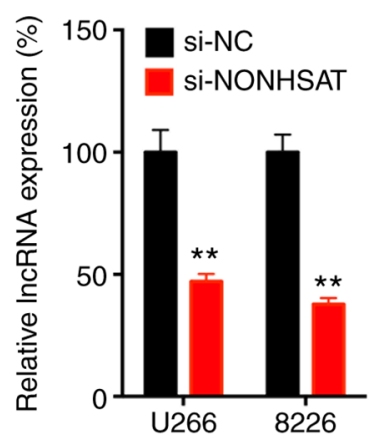

U266

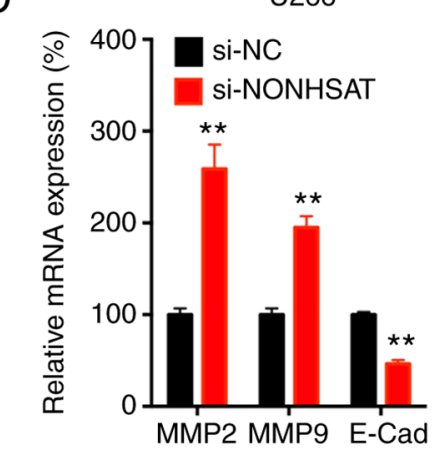

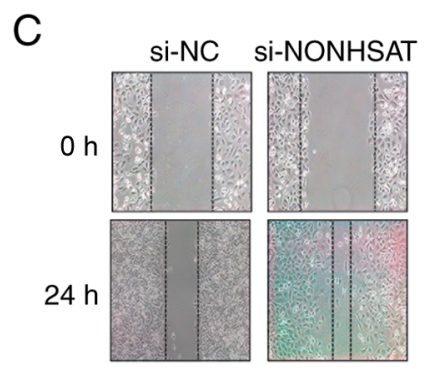

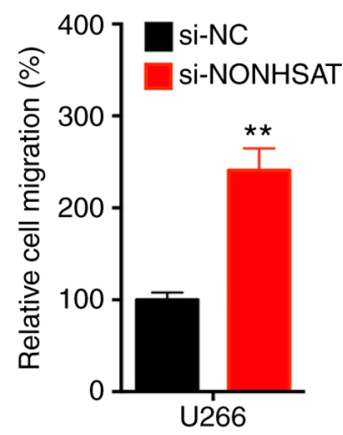

E

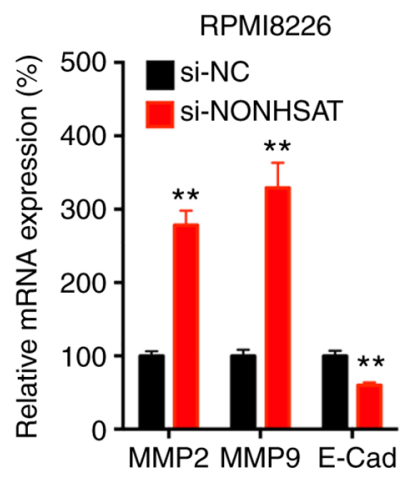

Figure 5. HDAC1 negatively regulates the expression of 1ncRNA NONHSAT113026 in MM cells. (A) Cells were transfected with si-NC or si-HDAC1 for $24 \mathrm{~h}$, the expression of lncRNA NONHSAT113026 was measured via RT-qPCR. (B) Cells were transfected with si-NC or si-lncRNA NONHSAT113026 for $24 \mathrm{~h}$, the expression of lncRNA NONHSAT113026 was measured. (C) U266 cells were transfected with si-NC or si-lncRNA NONHSAT113026 for $24 \mathrm{~h}$, cell migration was evaluated by wound healing assay. (D) U266 or (E) RPMI8226 cells were pre-transfected with si-NC or si-lncRNA NONHSAT113026 for $24 \mathrm{~h}$ and then expression of MMP2, MMP9 and E-Cad were measured via RT-qPCR. Data are presented as the mean \pm SD of three independent experiments. ** $\mathrm{P}<0.01$. HDAC1, histone deactylase 1; MM, multiple myeloma; si, small interfering; NC, negative control; lnc, long non-coding; E-Cad, E-cadherin.

the expression of MMP-2 and MMP-9, and decrease the expression of E-Cad, in both U266 (Fig. 5D) and RPMI8226 (Fig. 5E) cells. These results suggested that HDAC1 negatively regulated the expression of IncRNA-NONHSAT113026 in MM cells.

lncRNA NONHSAT113026 influences the HDAC1-regulated expression of Slug. The present study further investigated whether IncRNA NONHSAT113026 was involved in the HDAC1-regulated expression of Slug. The current data showed that knockdown of IncRNA-NONHSAT113026 significantly attenuated the si-HDAC1-suppressed mRNA expression of Slug in both U266 (Fig. 6A) and RPMI8226 (Fig. 6B) cells. Consistently, knockdown of lncRNA-NONHSAT113026 also reversed the si-HDAC1-suppressed protein expression of Slug in U266 (Fig. 6C) and RPMI8226 (Fig. 6D) cells. The mRNA stability assay revealed that knockdown of lncRNA-NONHSAT113026 significantly increased the mRNA stability of Slug in both U266 (Fig. 6E) and RPMI8226 (Fig. 6F) cells. Silencing of lncRNA-NONHSAT113026 also attenuated the si-HDAC1-increased expression of E-Cad in U266 cells (Fig.6G). These data indicated that lncRNA-NONHSAT113026 was involved in the HDAC1-regulated expression of Slug.

\section{Discussion}

The present study demonstrated that HDAC1 was increased in MM cells and tissues, and it regulated cell migration and increased the expression of MMPs in MM cells. It has been reported that chromatin state changes regulated by HDACs are closely associated with myeloma progression (31), and HDACs can regulate the expression levels of genes involved in myeloma cell proliferation (32). High levels of HDAC1 were also observed in MM cells compared with those in normal cells (33). In addition, increased expression of HDAC1 was also observed in prostate and breast cancer $(34,35)$. Previous reports also indicated that the HDAC inhibitors vorinostat and valproic acid (VPA) can decrease the migration ability of myeloma cells via increasing the expression of plasma membrane $\mathrm{Ca}^{2+}$ ATPase 4b (36). In addition, class I HDAC inhibitors, such as VPA and MS-275, can enhance the effects of chemotherapy on myeloma cells (33). Overall, these data confirmed the oncogenic roles of HDAC1 in myeloma progression, and indicated that targeted inhibition of HDAC1 may represent a potential approach to myeloma treatment.

The present study revealed that Slug was essential for the HDAC1-regulated migration of MM cells. Slug is a transcription factor that promotes the EMT of cancer cells $(37,38)$. It can also inhibit the expression of cell adhesion proteins, such as E-cadherin, to induce cell migration (37,39). High Slug expression in tumors is often associated with increasing cell proliferation, apoptosis and invasion. Increased expression of Slug has been observed in primary and metastatic myeloma (40). Furthermore, silencing of Slug can increase the radiosensitivity of myeloma cells in vitro (41). Expression of exogenous Slug in melanocytes and myeloma cells in vitro can stimulate the migration and invasion of MM cells (40). The present data indicated that knockdown of HDAC1 can 


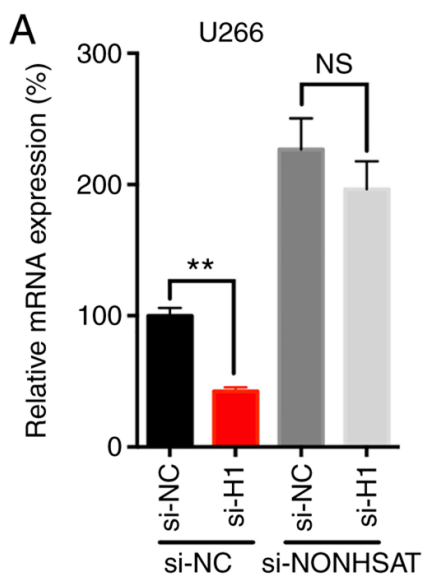

D

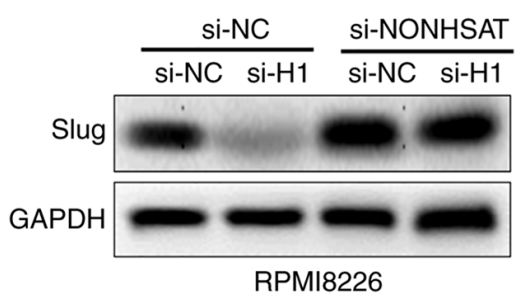

$\mathrm{F}$

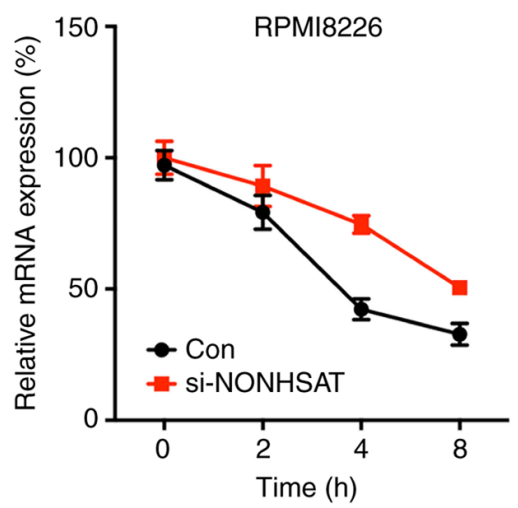

$\mathrm{B}$

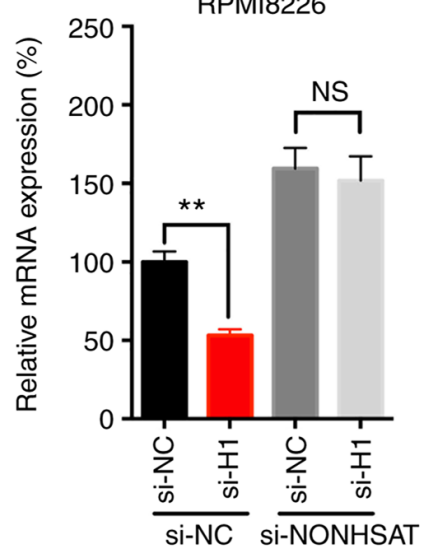

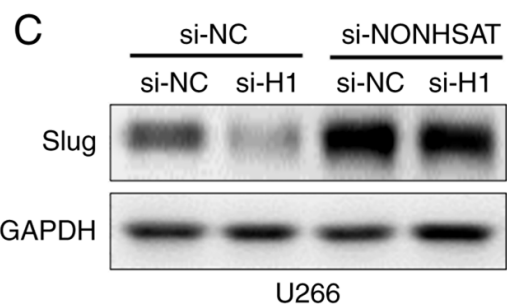

U266
$\mathrm{E}$

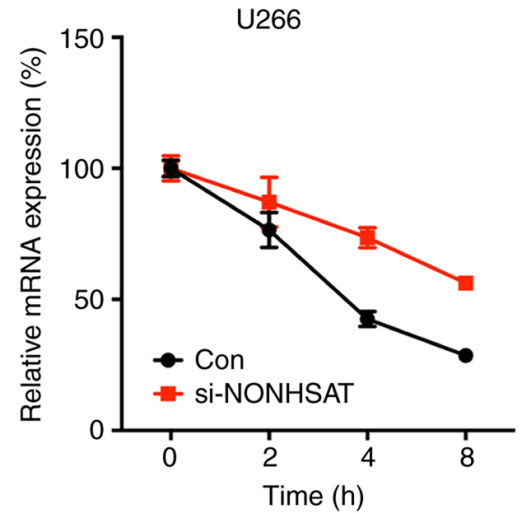

G

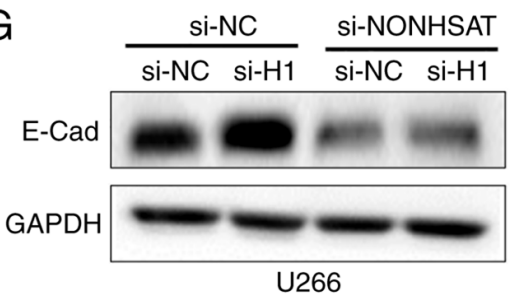

Figure 6. IncRNA NONHSAT113026 is involved in HDAC8-regulated expression of Slug. (A) U266 and (B) RPMI8226 cells were co-transfected with si-NC, si-HDAC1 and si-lncRNA NONHSAT113026 for $24 \mathrm{~h}$ before the mRNA expression of Slug was measured. (C) U266 and (D) RPMI8226 cells were co-transfected with si-NC, si-HDAC1 and si-lncRNA NONHSAT113026 for $24 \mathrm{~h}$ before the protein expression of Slug was measured. (E) U266 and (F) RPMI8226 cells were pre-transfected with si-NC or si-IncRNA NONHSAT113026 for $24 \mathrm{~h}$ and further treated with Act-D for indicated time periods before the mRNA of Slug was evaluated. (G) U266 cells were co-transfected with si-NC, si-HDAC1 and si-IncRNA NONHSAT113026 for $24 \mathrm{~h}$, the expression of E-Cad was checked. Data are presented as the mean \pm SD of three independent experiments. ${ }^{* *} \mathrm{P}<0.01$. NS, no significant; HDAC1, histone deactylase 1; MM, multiple myeloma; si, small interfering; NC, negative control; lnc, long non-coding.

suppress the expression of Slug, while overexpression of Slug can rescue si-HDAC1-inhibited cell migration. These findings confirmed the essential roles of Slug in the HDAC1-regulated progression of MM.

lncRNA NONHSAT113026-regulated mRNA stability is involved in the HDAC1-regulated expression of Slug. The present data showed that HDAC1 can regulate the mRNA stability of Slug, while it had no effect on its transcription or nuclear export. NONHSAT113026, which is localized at 6p12.3 and has a 7,682-bp RNA sequence encompassing four exons, was reported to be increased in renal cell carcinoma compared matched non-tumor tissues (42). It can directly bind to the 3'-UTR of Slug mRNA to trigger mRNA degradation via direct IncRNA-mRNA interactions that arise from hybridization of homologous sequences (30). Knockdown of HDAC1 can significantly increase the expression of NONHSAT113026; however, the detailed mechanisms need further investigation. In addition, a previous study also indicated that HDAC1 can recruit and antagonize Slug E-box binding (43); however, whether this also occurs in MM cells needs further research.

In conclusion, the present study demonstrated that HDAC1 was upregulated in MM cells and tissues, and targeted inhibition of HDAC1 suppressed the migration of MM cells via downregulation of Slug. Mechanistic studies indicated that si-HDAC1 can decrease mRNA stability via upregulation of lncRNA-NONHSAT113026. It should be noted that the expression of HDAC1 was increased in cultured cell lines. Futur studies should continue to isolate and culture patients' primary 
MM cells to validate the present conclusion. The current findings indicated that targeted inhibition of the HDAC1/Slug axis may represent a potential therapeutic option for patients with MM.

\section{Acknowledgements}

Not applicable.

\section{Funding}

The present study was supported by Fourth Pilot-Research Program for Human Space Flight (Grant/Award no. 020106).

\section{Availability of data and materials}

The datasets used and/or analyzed during the current study are available from the corresponding author on reasonable request.

\section{Ethics approval and consent to participate}

Not applicable.

\section{Patient consent for publication}

Not applicable.

\section{Authors' contributions}

LZ,JL, ML, MH and YZ conceived and designed the study. LZ, $\mathrm{JL}$ and $\mathrm{AZ}$ acquired the data. ML and $\mathrm{YZ}$ analyzed and interpreted the data. LZ and JL wrote and revised the manuscript. The authenticity of the raw data has been assessed by all authors. All authors read and approved the final manuscript.

\section{Competing interests}

The authors declare that they have no competing interests.

\section{References}

1. Landgren $\mathrm{O}$ and Morgan GJ: Biologic frontiers in multiple myeloma: From biomarker identification to clinical practice. Clin Cancer Res 20: 804-813, 2014.

2. Reisenbuckler C: Multiple myeloma and diagnostic imaging. Radiol Technol 85: 391-413, 2014.

3. Orlowski RZ: Why proteasome inhibitors cannot ERADicate multiple myeloma. Cancer Cell 24: 275-277, 2013.

4. McCullough KB, Hobbs MA, Abeykoon JP and Kapoor P: Common adverse effects of novel therapies for multiple myeloma (MM) and their management strategies. Curr Hematol Malig Rep 13: 114-124, 2018.

5. Naymagon L and Abdul-Hay M: Novel agents in the treatment of multiple myeloma: A review about the future. J Hematol Oncol 9: $52,2016$.

6. Ria R, Reale A and Vacca A: Novel agents and new therapeutic approaches for treatment of multiple myeloma. World J Methodol 4: 73-90, 2014

7. Varga C, Laubach J, Hideshima T, Chauhan D, Anderson KC and Richardson PG: Novel targeted agents in the treatment of multiple myeloma. Hematol Oncol Clin North Am 28: 903-925, 2014.

8. Kelly T, Børset M, Abe E, Gaddy-Kurten D and Sanderson RD: Matrix metalloproteinases in multiple myeloma. Leuk Lymphoma 37: 273-281, 2000.
9. Takimoto M, Ogawa K, Kato Y, Saito T, Suzuki T, Irei M, Shibuya Y, Suzuki Y, Kato M, Inoue Y, et al: Close relation between 14q32/IGH translocations and chromosome 13 abnormalities in multiple myeloma: A high incidence of 11q13/CCND1 and 16q23/MAF. Int J Hematol 87: 260-265, 2008.

10. Largo C, Saéz B, Alvarez S, Suela J, Ferreira B, Blesa D, Prosper F, Calasanz MJ and Cigudosa JC: Multiple myeloma primary cells show a highly rearranged unbalanced genome with amplifications and homozygous deletions irrespective of the presence of immunoglobulin-related chromosome translocations. Haematologica 92: 795-802, 2007.

11. Bergsagel PL and Kuehl WM: Chromosome translocations in multiple myeloma. Oncogene 20: 5611-5622, 2001.

12. Issa ME, Takhsha FS, Chirumamilla CS, Perez-Novo C, Vanden Berghe $\mathrm{W}$ and Cuendet $\mathrm{M}$ : Epigenetic strategies to reverse drug resistance in heterogeneous multiple myeloma. Clin Epigenetics 9: 17, 2017.

13. Liang X, Deng M, Zhang C, Ping F, Wang H, Wang Y, Fan Z, Ren X, Tao X, Wu T, et al: Combined class I histone deacetylase and $\mathrm{mTORC} 1 / \mathrm{C} 2$ inhibition suppresses the initiation and recurrence of oral squamous cell carcinomas by repressing SOX2. Cancer Lett 454: 108-119, 2019.

14. Jeon YJ, Ko SM, Cho JH, Chae JI and Shim JH: The HDAC inhibitor, panobinostat, induces apoptosis by suppressing the expresssion of specificity protein 1 in oral squamous cell carcinoma. Int J Mol Med 32: 860-866, 2013.

15. Zhu P, Martin E, Mengwasser J, Schlag P, Janssen KP and Göttlicher M: Induction of HDAC2 expression upon loss of APC in colorectal tumorigenesis. Cancer Cell 5: 455-463, 2004.

16. Barneda-Zahonero B and Parra M: Histone deacetylases and cancer. Mol Oncol 6: 579-589, 2012.

17. Garcia-Guerrero E, Götz R, Doose S, Sauer M, Rodríguez-Gil A, Nerreter T, Kortüm KM,Pérez-Simón JA, Einsele H,Hudecek M and Danhof S: Upregulation of CD38 expression on multiple myeloma cells by novel HDAC6 inhibitors is a class effect and augments the efficacy of daratumumab. Leukemia 35: 201-214, 2021.

18. Zhou Y, Liu X, Xue J, Liu L, Liang T, Li W, Yang X, Hou X and Fang H: Discovery of peptide boronate derivatives as histone deacetylase and proteasome dual inhibitors for overcoming bortezomib resistance of multiple myeloma. J Med Chem 63: 4701-4715, 2020

19. Sun X, Xie Y, Sun X, Yao Y, Li H, Li Z, Yao R and Xu K: The selective HDAC6 inhibitor nexturastat A induces apoptosis, overcomes drug resistance and inhibits tumor growth in multiple myeloma. Biosci Rep 39: BSR20181916, 2019.

20. Mithraprabhu S, Kalff A, Chow A, Khong T and Spencer A: Dysregulated class I histone deacetylases are indicators of poor prognosis in multiple myeloma. Epigenetics 9: 1511-1520, 2014.

21. Alanazi F, Kwa FAA, Burchall G and Jackson DE: New generation drugs for treatment of multiple myeloma. Drug Discov Today 25: 367-379, 2020.

22. Hadley M, Noonepalle S, Banik D and Villagra A: Functional analysis of HDACs in tumorigenesis. Methods Mol Biol 1983: 279-307, 2019.

23. Chen Y,Zhao H, Li H, Feng X, Tang H, Qiu C, Zhang J and Fu B: LINC01234/MicroRNA-31-5p/MAGEA3 axis mediates the proliferation and chemoresistance of hepatocellular carcinoma cells. Mol Ther Nucleic Acids 19: 168-178, 2020.

24. Furuyama T, Nakazawa T, Nakano I and Mori N: Identification of the differential distribution patterns of mRNAs and consensus binding sequences for mouse DAF-16 homologues. Biochem J 349: 629-634, 2000.

25. Zhao Y, Liu Y, Lin L, Huang Q, He W, Zhang S, Dong S, Wen Z, Rao J, Liao W and Shi M: The lncRNA MACC1-AS1 promotes gastric cancer cell metabolic plasticity via AMPK/Lin 28 mediated mRNA stability of MACC1. Mol Cancer 17: 69, 2018.

26. Brabletz T, Kalluri R, Nieto MA and Weinberg RA: EMT in cancer. Nat Rev Cancer 18: 128-134, 2018.

27. Chaffer CL, San Juan BP, Lim E and Weinberg RA: EMT, cell plasticity and metastasis. Cancer Metastasis Rev 35: 645-654, 2016.

28. Quinn JJ and Chang HY: Unique features of long non-coding RNA biogenesis and function. Nat Rev Genet 17: 47-62, 2016.

29. Yao RW, Wang Y and Chen LL: Cellular functions of long noncoding RNAs. Nat Cell Biol 21: 542-551, 2019.

30. Pu Y, Dong Z, Xia Y, Zhang M, Song J, Han J and Liu H: LncRNA NONHSAT113026 represses renal cell carcinoma tumorigenesis through interacting with $\mathrm{NF}-\kappa \mathrm{B} / \mathrm{p} 50$ and SLUG. Biomed Pharmacother 118: 109382, 2019. 
31. Al Emran A, Marzese DM, Menon DR, Stark MS, Torrano J, Hammerlindl H, Zhang G, Brafford P, Salomon MP, Nelson N, et al: Distinct histone modifications denote early stress-induced drug tolerance in cancer. Oncotarget 9: 8206-8222, 2017.

32. Yeon M, Kim Y, Jung HS and Jeoung D: Histone deacetylase inhibitors to overcome resistance to targeted and immuno therapy in metastatic melanoma. Front Cell Dev Biol 8: 486, 2020.

33. Krumm A, Barckhausen C, Kücük P, Tomaszowski KH, Loquai C, Fahrer J, Krämer OH, Kaina B and Roos WP: Enhanced histone deacetylase activity in malignant melanoma provokes RAD51 and FANCD2-triggered drug resistance. Cancer Res 76: 3067-3077, 2016.

34. Gameiro SR, Malamas AS, Tsang KY, Ferrone S and Hodge JW: Inhibitors of histone deacetylase 1 reverse the immune evasion phenotype to enhance T-cell mediated lysis of prostate and breas carcinoma cells. Oncotarget 7: 7390-7402, 2016.

35. Tang Z, Ding S, Huang H, Luo P, Qing B, Zhang S and Tang R: HDAC1 triggers the proliferation and migration of breast cancer cells via upregulation of interleukin-8. Biol Chem 398: 1347-1356, 2017.

36. Hegedüs L, Padányi R, Molnár J, Pászty K, Varga K, Kenessey I, Sárközy E, Wolf M, Grusch M, Hegyi Z, et al: Histone deacetylase inhibitor treatment increases the expression of the plasma membrane $\mathrm{Ca}^{2+}$ pump PMCA4b and inhibits the migration of melanoma cells independent of ERK. Front Oncol 7: 95, 2017.

37. Wang Y, Shi J, Chai K, Ying X and Zhou BP: The role of snail in EMT and tumorigenesis. Curr Cancer Drug Targets 13: 963-972, 2013.
38. Ye X, Brabletz T, Kang Y, Longmore GD, Nieto MA, Stanger BZ, Yang $\mathrm{J}$ and Weinberg RA: Upholding a role for EMT in breast cancer metastasis. Nature 547: E1-E3, 2017.

39. Wang $\mathrm{Z}$ and Liu C: MiR-153 regulates metastases of gastric cancer through Snail. Tumor Biol 37: 15509-15515, 2016.

40. Shirley SH, Greene VR, Duncan LM, Torres Cabala CA, Grimm EA and Kusewitt DF: Slug expression during melanoma progression. Am J Pathol 180: 2479-2489, 2012.

41. Arienti C, Tesei A, Carloni S, Ulivi P, Romeo A, Ghigi G, Menghi E, Sarnelli A, Parisi E, Silvestrini R and Zoli W: SLUG silencing increases radiosensitivity of melanoma cells in vitro. Cell Oncol (Dordr) 36: 131-139, 2013.

42. Liu H, Chen P, Jiang C, Han J, Zhao B, Ma Y and Mardan M: Screening for the key lncRNA targets associated with metastasis of renal clear cell carcinoma. Medicine (Baltimore) 95: e2507, 2016.

43. Lin CW, Wang LK, Wang SP, Chang YL, Wu YY, Chen HY, Hsiao TH, Lai WY, Lu HH, Chang YH, et al: Daxx inhibits hypoxia-induced lung cancer cell metastasis by suppressing the HIF-1 $\alpha /$ HDAC1/Slug axis. Nat Commun 7: 13867, 2016.

(i) () This work is licensed under a Creative Commons Attribution-NonCommercial-NoDerivatives 4.0 International (CC BY-NC-ND 4.0) License. 